\title{
EXTENSION OF THE OPTICAL REFERENCE FRAME:SPACE BASED
}

\author{
S. RÖSER \\ Astronomisches Rechen-Institut, s19@ix.urz.uni-heidelberg.de \\ Mönchhofstr. 12-14, D-69120 Heidelberg, Germany
}

\section{Introduction}

At its 23rd General Assembly, Kyoto 1997, the IAU decided that the International Celestial Reference Frame (ICRF) should be defined by the positions of 608 compact extragalactic radio sources (Ma \& Feissel 1997), with the Hipparcos Catalogue (ESA 1997) being its practical realization at optical wavelengths. Although the Hipparcos instrument was unable to observe the defining sources of the ICRF directly in the optical regime, it is linked to the ICRF via a number of auxiliary observations (Kovalevsky et al. 1997). The Hipparcos Catalogue is the first celestial reference frame which is constructed from observations not made from the surface of the Earth.

Hipparcos represents a major break-through in astrometry at the end of this century and it increases the number of stars in the reference system by a factor of 30 compared to its predecessor, the FK5 (Fricke et al. 1988). However, the 120000 reference stars of Hipparcos are mostly in the range between the 7th and 9th magnitudes. This fact asks for extension to fainter magnitudes and more objects. The short observational period of Hipparcos determines the accuracy with which proper motions could be determined. At its mean epoch, 1991.25, the Hipparcos frame coincides with the ICRF to better than 0.6 mas in all three axes, and deviates from inertiality by not more than $0.25 \mathrm{mas} / \mathrm{yr}$ in all three axes (ESA 1997). Mainly, because of the proper motions' accuracy, the coincidence with ICRF will be not better than 5 mas in 2010. This asks for maintenance and improvement of the Hipparcos system.

\section{Space missions, overview}

Hipparcos has proven that space-based astrometry is, in many aspects, superior to measurements from ground. Consequently, Hipparcos is not the end of the road, but rather the beginning of a new and exciting branch of astrometry. One of the important features of Hipparcos is one-dimensional observations on a great circle using the closure conditions after each revolution. It is therefore quite obvious to use the improved resolution and accuray of an interferometer in space for further progress. And it is for this reason that all the proposed new space missions select a Fizeau interferometer as the instrument.

GAIA (Global Astrometric Interferometer for Astrophysics) (Lindegren \& Perryman 1996) is the most ambitious of all the new missions. According to latest estimates it should be able to observe $10^{9}$ objects brighter than $\mathrm{V}=20$ and is aiming at an accuracy of $10 \mu$ as in position and parallax and $20 \mu \mathrm{as} / \mathrm{yr}$ in proper motion at $\mathrm{V}=15$. GAIA will be able to directly observe the defining sources of ICRF - mostly 18th magnitude - which will limit the deviation from an inertial frame to less than $10 \mu \mathrm{as} / \mathrm{yr}$. GAIA is proposed as an ESA mission with a possible launch in 2009 and final results in 2018 at the earliest.

FAME (Fizeau Astrometric Mapping Explorer) (Sejdelmann et al. 1996) is under study at the USNO and CfA, Cambridge, USA. It is aiming at an accuracy of $50 \mu$ as at $\mathrm{V}=10$. A possible launch date for FAME could be in the first half of the next decade.

LIGHT (Light Interferometer satellite for the studies of Galactic Halo Tracers) (Yoshizawa et al. 1997) is proposed by NAO (Japan) and others. It will have an interferometer onboard operating in visual and an infrared channels (at $2.4 \mu \mathrm{m}$ ). Detailed performance analysis for LIGHT is not available at the time of writing: the reader is referred to Yoshizawa et al. 1997. Launch of LIGHT could be between 2007 and 2010 . 
DIVA (Double Interferometer for Visual Astrometry) (Röser et al. 1997a), see also Röser et al. $1997 \mathrm{~b}$ in this volume, is a mission proposed to the German Aerospace Center (DLR). It has a good chance of being selected as the next German small-satellite mission with a launch in 2003.

\section{The DIVA mission}

DIVA (see Fig. 1) is a Fizeau interferometer on a small satellite of about $1 \mathrm{~m}$ diameter and a mass of $100 \mathrm{~kg}$. It has two small interferometer apertures of $5 \times 5 \mathrm{~cm}^{2}$ each, separated by a baselength of $10 \mathrm{~cm}$. A beam combiner allows quasi-simultaneous observations of two lines of sight separated by $60^{\circ}$. The limiting magnitude of DIVA is about $V=15$ for spectral types earlier than Mo, but drops to about $V=17.5$ for stars later than M5. At least 4 million stars will be measured, giving a complete sky survey down to $V=12.5$. After two years of mission the astrometric accuracy of 10 th magnitude stars will be 0.35 mas for positions and parallaxes, and 0.5 mas/yr for proper motions.

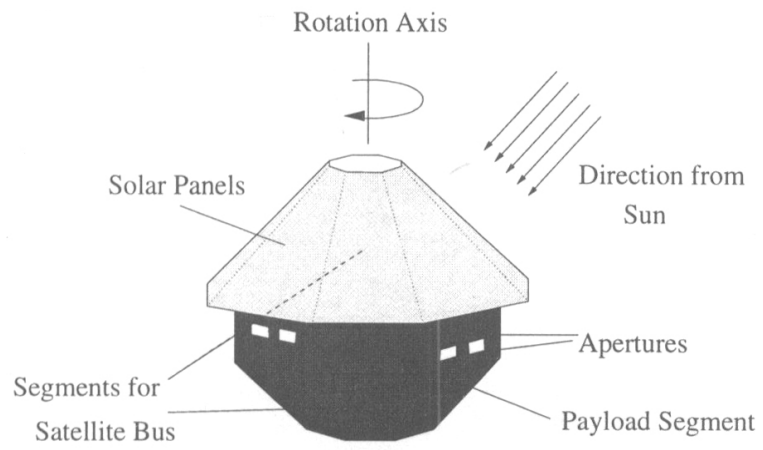

Figure 1. A schematic view of the DIVA spacecraft.

DIVA will be able to observe a few QSOs and AGNs down to its limiting magnitude. Estimates for $3 \mathrm{C} 273$ show that an accuracy of 2.6 mas for the position and 3.6 mas/yr for the proper motion are to be expected for this quasar. So, the link of the DIVA system to ICRS must be performed in a similar manner to Hipparcos, with most emphasis to the VLBI observations. Supposing that at least the same number of radio stars as with Hipparcos will be observed, and considering the improved accuracy of the DIVA observations, a coincidence with ICRF of \pm 0.3 mas can be expected for the mean epoch of DIVA observations around mid-2004. This, together with the link of Hipparcos to ICRF, yields a new representation of the ICRF in the optical regime which does not deviate from inertial by more than $50 \mu \mathrm{as} / \mathrm{yr}$.

In summary, we can state, that a small, cheap and fast mission like DIVA will be sufficient to improve the Hipparcos system and extend it to fainter and 40 times more stars. This improved system can be available from 2007 onwards, provided that the implementation of the DIVA mission can be accomplished as planned.

\section{References}

ESA (1997) The Hipparcos and Tycho Catalogues. European Space Agency, SP-1200

Fricke, W., Schwan H, Lederle, T. et al. (1988). Fifth Fundamental Catalogue (FK5). Part I. The Basic Fundamental Stars. Veröff. Astron. Rechen.Institut Heidelberg, Nr.32.

Kovalevsky, J. et al. (1997) The Hipparcos Catalogue as a realisation of the extragalactic reference system, $A \& A A$ 323,620

Lindegren, L. \& Perryman, M.A.C. (1997) GAIA: Global Astrometric Interferometer for Astrophysics, A $\& A S$ 116, 579

Ma, C. \& Feissel, M. (1997) Definition and Realization of the International Celestial Reference System by VLBI Astrometry of Extragalactic Objects, IERS Technical Note $\mathbf{2 3}$

Röser, S., Bastian, U., de Boer, K.S., Høg, E., Röser, H.P, Schalinski, C., Schilbach, E., de Vegt, Ch., Wagner, S. (1997a) DIVA - Towards Microarcsecond Global Astronetry, ESA-SP-402, (in press)

Röser, S., Bastian, U., de Boer, K.S., Høg, E., Schilbach, E., de Vegt, Ch., Wagner, S. (1997b) DIVA - a small satellite for glolal astrometry and photometry, (this volume). 
Seidelmann, P.K. et al. (1996) A Fizeau Optical Interferometer Astrometric Satellite, in: Perryman, M.A.C. \& van Leeuwen, F. (eds.), ESA-SP-379, 187

Yoshizawa, M., Sato, K., Nishikawa, J., Fukushima, T., Miyamoto, M. (1997) Optical/Infrared Astrometric Satellite Project LIGHT, ESA-SP-402, (in press) 